\title{
Uncoded Multiview Video Signal Transmission in Pre-ZF Equalization Aided MIMO Wireless Communication System
}

\author{
Shammi Farhana Islam ${ }^{1}$, Shaikh Enayet Ullah ${ }^{2}$ and Md. Mahbubar Rahman ${ }^{3}$ \\ ${ }^{1}$ Department of Material Science and Engineering, Rajshahi University, Rajshahi- \\ 6205, Bangladesh \\ ${ }^{2}$ Department of Applied Physics and Electronic Engineering, Rajshahi University, \\ Rajshahi-6205, Bangladesh \\ ${ }^{3}$ Department of Applied Physics, Electronics and Communication Engineering, \\ Islamic University, Kushtia-7003, Bangladesh \\ shammi.farhana@gmail.com,enayet_apee@ru.ac.bdandmahbublv@yahoo.com
}

\begin{abstract}
This research study has been made on the quality assessment of the multiview video signal transmitted in a correlated MIMO fading channel. In matlab based simulated system, pre- zero forcing channel Equalization/ precoding, two-dimensional nonlinear Median filtering for impulsive noise reduction schemes have been implemented under scenario of adding no additional redundant data for desired video signal protection. It is noteacable from such study that themultiview video signal with quite reasonably acceptable image quality is retrieved.
\end{abstract}

Keywords: multiview video, Uncoded transmission, Pre-ZF equalization, signal to noise ratio $(S N R)$

\section{Introduction}

With technological development in multimedia, a great emphasis is being given on three-dimensional (3D) video. The three-dimensional (3D) video is increasingly considered as the next major innovation in video technology, with the goal to provide a leap forward in quality of experience. The 3D video are represented as stereoscopic 3D (S3D) video, multi-view video (MVV), video-plus-depth, multi-view video-plus-depth (MVD) and layered depth video (LDV). In Multiview Video (MVV) representation, several texture videos are acquired in a synchronous manner by a system of cameras capturing the same scene from varying viewpoints. The MVV is characterized by significant inter-view statistical redundancies. The views in MVV can be independently encoded (i.e., simulcast), or jointly encoded by taking advantage of the correlations existing amongst the views. The MVV is especially suited for emerging auto-stereoscopic displays, which require a large number of views with preservation of the full resolution of the video sequence. The major shortcoming of the representation is that, even when using an efficient coding scheme such as MVC, the bit rate essentially grows linearly with the number of encoded views $[1,2]$.

In 2014, Hao Cui and et. al., made a comprehensive research study on robust uncoded video transmission over wireless fast fading channel under consideration of availability of statisticalchannel state information (CSI) at the transmitter side. Under such work, it was observed that an increase in channel diversity for high priority (HP) data is essential to improve the robustness of video transmissionin fading channels. With utilization of the noise and loss resilient nature of video, it is found to have possibility to design a more robust system by re-allocating the power and channel uses among HP and LP (low priority) data [3]. 
The present study is not discriminating video data into HP and LP forms. The captured multiview video (MVV) data will be sent up through the uncorrelated MIMO without implementation of channel coding scheme viz. addition of redundant data for protecting video data.

\section{Signal Processing Techniques}

In this paper, various signal processing techniques outlined below have been used.

\subsection{Pre-ZF Equalization}

The frame sequential video frames are processed for pixel contamination with salt and paper noise, integer to binary bit conversion and eventually converted into digitally modulated symbol vector $\mathbf{X}$. The pre- ZF Equalizer weight matrix

$\mathrm{W}_{\mathrm{ZF}} \in \mathrm{C}^{4 \times 4}$ can be written as

$$
\mathbf{W}_{\text {ZF }}=\beta \mathbf{H}^{-1}
$$

where $\beta$ is a constant to meet up the total transmitted power constraint after preequalization and is given by

$$
\beta=\sqrt{\frac{4}{\operatorname{Tr}\left(\mathbf{H}^{-1}\left(\mathbf{H}^{-1}\right)^{\mathbf{H}}\right)}}
$$

and the $\mathrm{H} \in \mathrm{C}^{4 \times 4}$ is spatially uncorrelated MIMO channel matrix.

The ZF precoded symbol vector $\overline{\mathrm{x}}=\mathrm{w}_{\mathrm{ZF}} \mathrm{X}$ and the received signal $\mathrm{Y}$ in terms of channel matrix $\mathrm{H}$, transmitted signal $\overline{\mathrm{X}}$ and AWGN noise $\mathrm{N}$ can be written as

$$
\mathbf{Y}=\mathbf{H W}_{\mathbf{Z F}} \mathbf{X}+\mathbf{N}=\mathbf{H} \overline{\mathbf{X}}+\mathbf{N}
$$

The detected digitally modulated symbol vector $\tilde{\mathrm{x}}$ can be written as[4]

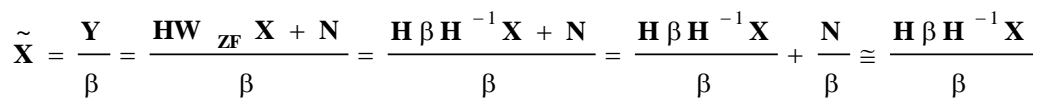

\subsection{2-D Median Filtering}

The 2-D Median Filter is a non- linear filter or order-static filter used for salt and pepper noise reduction. In such scheme, $3 \times 3$ neighborhood window is used. This filter simply sorts all pixel values within the window, finds the median value, and replaces the original pixel value with the median value [5].

\section{System Description}

The simulated uncoded multiview video signal processing system with implementation of ZF pre equalization based precoding scheme is depicted in Figure 1. 


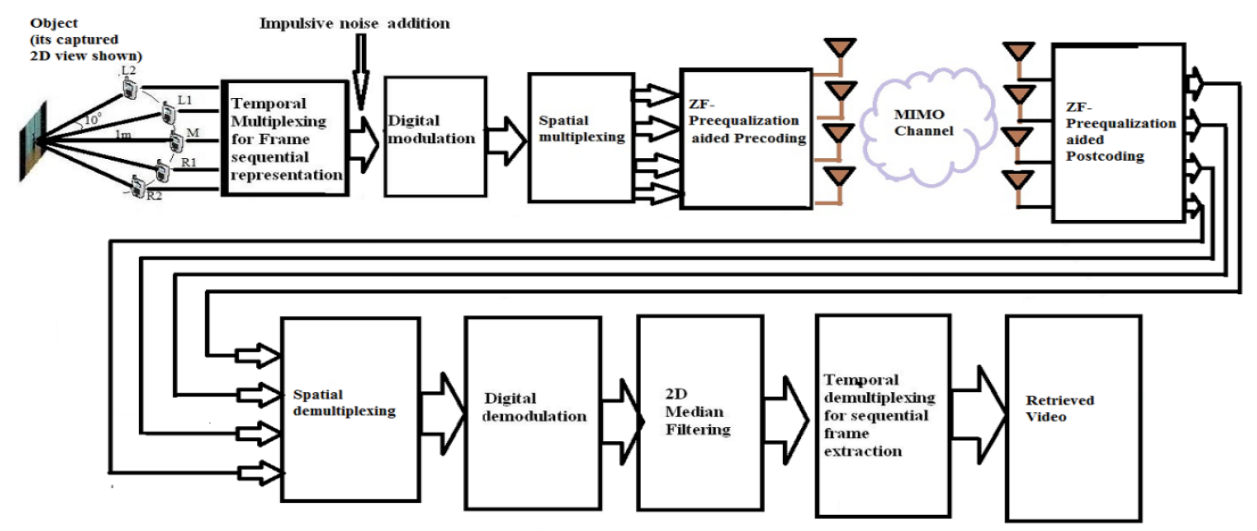

\section{Figure 1. Simplified bLock Diagram of Uncoded pre-ZF Equalization Aided Multiview Video Signal Processing MIMO System}

The videos of a fixed object are captured by Sony Xperia $\mathrm{Z}$ smart phone from five locations( two left:L1,L2,one middle :M, and two right: R1 and R2) situated in a arc path maintaining a distance of 1 meter from the object. The angular displacement between two consecutive locations is 10 degree. The time duration of each video capturing is 10 seconds. The captured video frames are temporally multiplexed for frame sequential representation. The multiplexed video frames are contaminated with salt and pepper noise and are digitally modulated using 64QAM [6]. The digitally modulated complex symbols are spatially multiplexed, precoded and sent up from each of the four transmitting antennas. At the receiver, postcoding scheme is implemented. The extracted complex modulated symbols are spatially demultiplexed, digitally demodulated and filtered for salt and pepper noise reduction. The impulsive noise free video frames are temporally demultiplexed to retrieve transmitted multiview video.

\section{Result and Discussion}

We have conducted computer simulations using MATLAB R2014a to evaluate the quality of the multiview video signal transmitted in an uncoded system based on the parameters given in Table 1. It is assumed that the channel state information (CSI) of the MIMO fading channel is available at the receiver and the fading process is approximately constant during the whole frame sequential multiview transmission

Table 1. Summary of the Simulated Model Parameters

\begin{tabular}{|l|c|}
\hline $\begin{array}{l}\text { No. of location of } \\
\text { video Capturing }\end{array}$ & 5 \\
\hline $\begin{array}{l}\text { No. of video frames } \\
\text { considered at each } \\
\text { location }\end{array}$ & 5 \\
\hline $\begin{array}{l}\text { Antenna } \\
\text { Configuration }\end{array}$ & $4 \times 4$ \\
\hline $\begin{array}{l}\text { No of video } \\
\text { frames/second }\end{array}$ & 30 \\
\hline Video frame size & 1088 pixels(height) $\times 1920$ pixels(width) \\
\hline Digital Modulation & R4QAM \\
\hline Fading channel & Rayleigh Fading \\
\hline Noise type & Gaussian and Impulsive(salt and pepper) \\
\hline $\begin{array}{l}\text { Signal to noise ratio } \\
\text { (SNR) }\end{array}$ & \\
\hline
\end{tabular}


In Figure 2, a total number of 25 video frames (60th, 120th, 180th, 240th and 300th at each of five locations L2, L1, M, R1 and R2) have been presented. In Figure 3, Impulsive (salt and pepper) noise contaminated video frames are shown. Each color video frame consists of Red, Green and Blue components with 1088 pixels $\times 1920$ pixels size and its $4 \%$ viz. $\mathbf{8 3 5 5 8}$ pixels out of $\mathbf{2 0 8 8 9 6 0}$ pixels are contaminated with impulsive noise. In Figure 4 through Figure 8, it is observable that in all cases with consideration of $4 \%$ of the total pixels of each color video frame contamination with Salt and pepper noise, the estimated bit error rate is around $11 \%$ in case of extreme hostile situation where, the transmitted signal power is identical with additive white Gaussian noise power $(\mathrm{SNR}=0 \mathrm{~dB})$. At reasonably considered $\mathrm{SNR}$ value of $5 \mathrm{~dB}$, the estimated bit error rate is found to be within the range of $5.13 \%$ to $5.63 \%$. In Figure 9, it is quite understandable theuncoded system performance for a typically assumed 127th video frame captured with smartphone located at the central line is satisfactory. In such case, the number of pixels contaminated with salt and pepper noise is also 83558. In Figure 10, histograms of captured RGB to Gray converted 127th video frame, its noise contaminated and retrival scenario are presented. The histograms are indicative of pixel intensity values( 0 to 255)and the absence of intensity values in the upper range: 200-255 confirms that the captured video frame is not bright. Most of the pixel intensity values lie in the range of 75 to 150 .

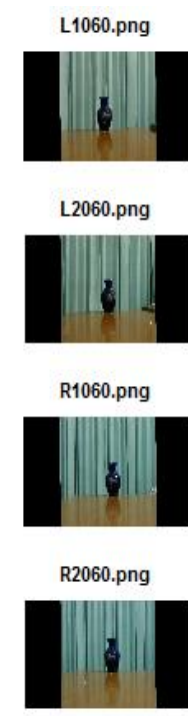

M060.png

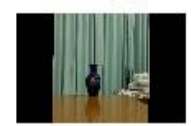

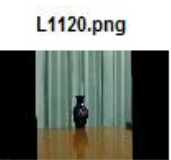

L2120.png

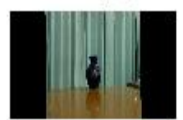

R1120.png

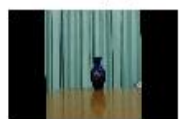

R2120.png

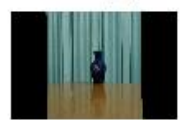

M120.png

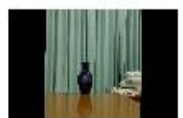

L1180.png

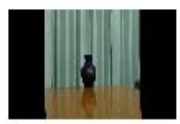

L2180.png

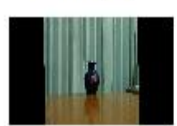

R1180.png

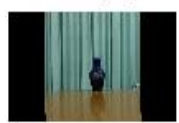

R2180.png

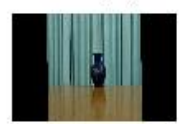

M180.png

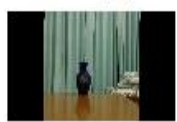

L1240.png

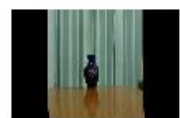

L2240.png

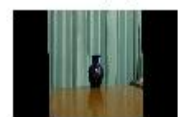

R1240.png

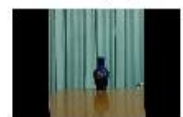

R2240.png

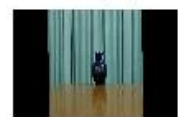

M240.png

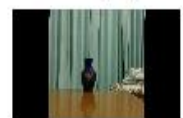

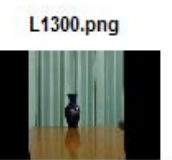

L2300.png

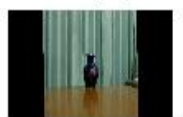

R1300.png

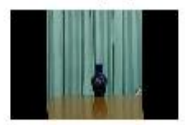

R2300.png

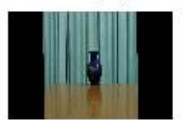

M300.png

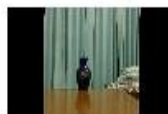

Figure 2. Captured Five Selected Video Frames $(60$ th, 120th, 180th, 240th And 300th) at each of Five Locations L2, L1, M, R1 And R2 


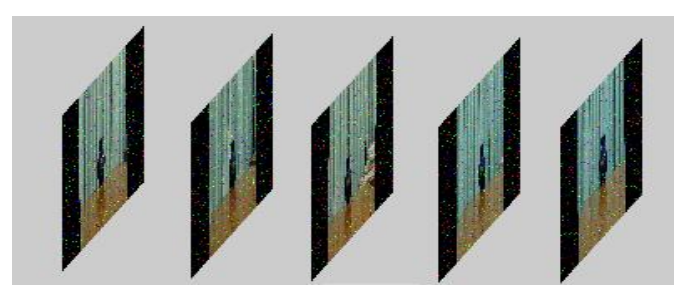

(a) 60

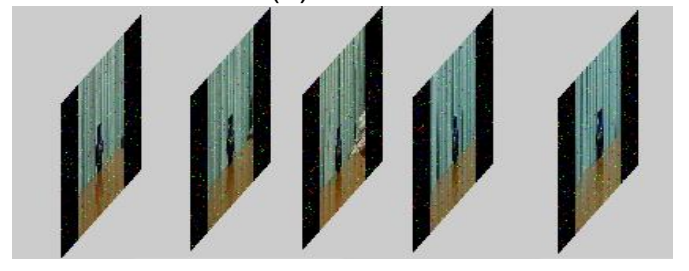

(c) 180

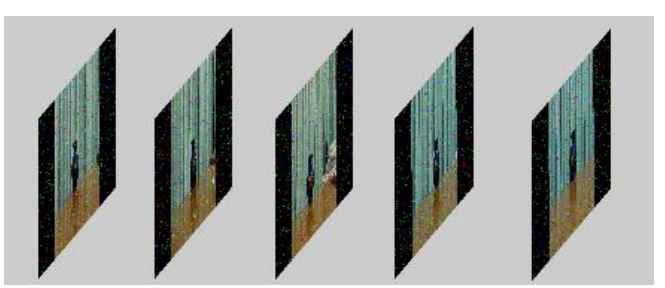

(b) 120

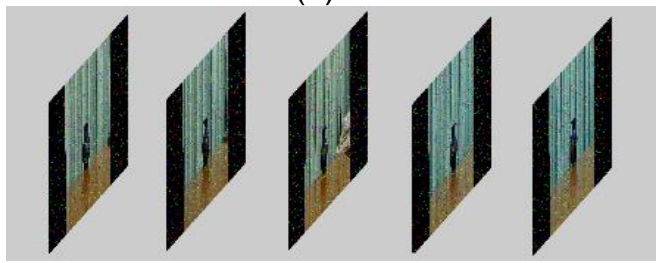

(d) 240

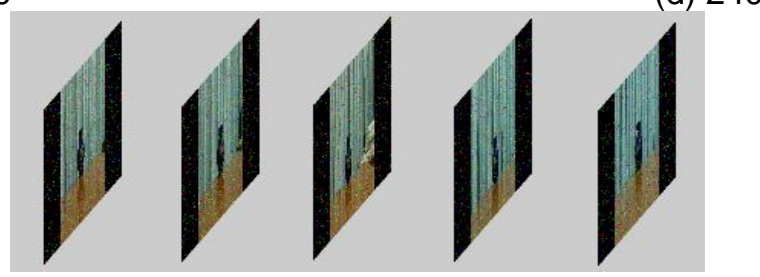

(e) 300

Figure 3. Salt and Pepper Noise Contaminated Frame Sequential Video Frames at Five Locations: L2, L1, M, R1 and R2(From Left to Right); (A): 60th Video Frames,(B)120th Video Frames,(C)180th Video Frames,(D)240th Video Frames and (E)300th Video Frames

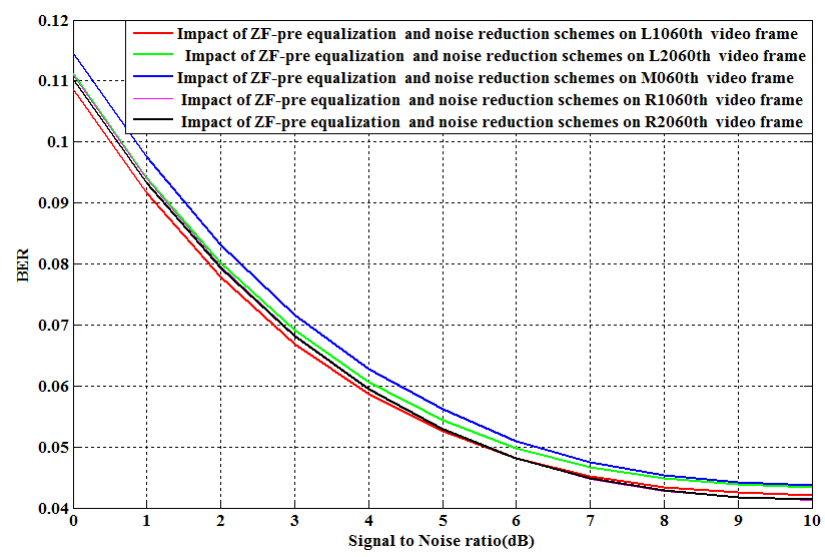

Figure 4. BER Performance of Pre-ZF Equalization Based MIMO System with Implementation of 2D Median Filtering and 64 QAM Digital Modulation Schemeson Frame Sequential Video Frames (60th Frame of Each of Five Locations L2, L1, M, R1 and R2) 


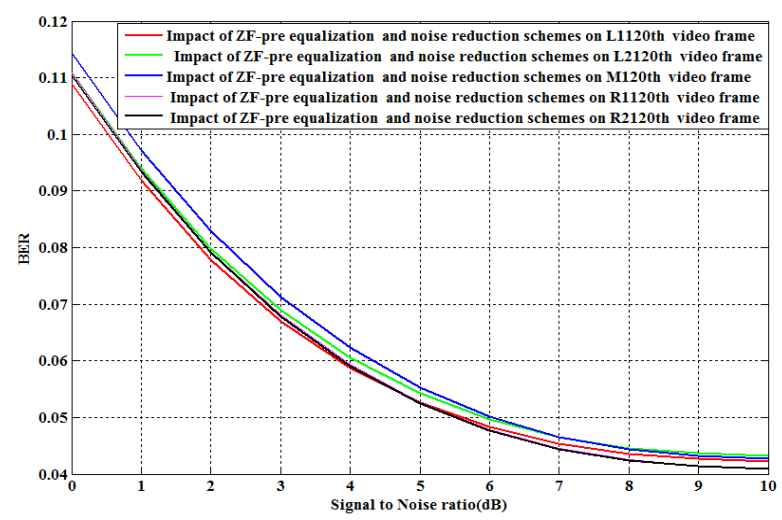

Figure 5. BER Performance of Pre-ZF Equalization Based MIMO System with Implementation of 2D Median Filtering and 64 AM Digital Modulation Schemes on Frame Sequential Video Frames (120th Frame of Each of Five Locations L2, L1, M, R1 and R2)

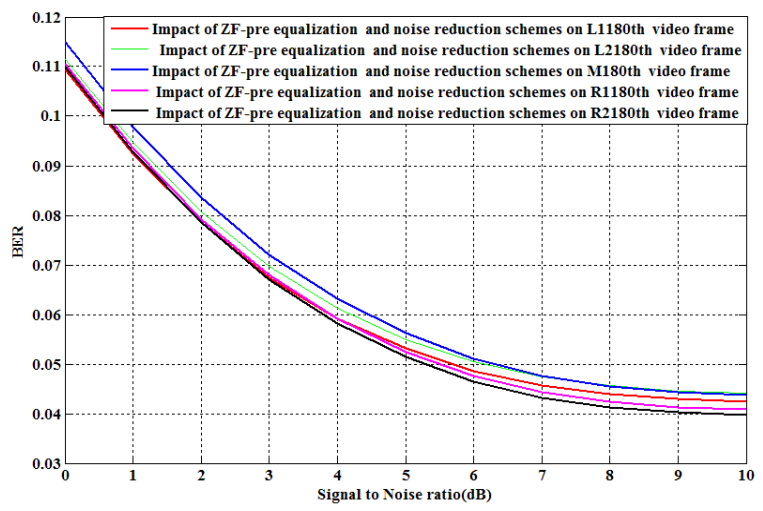

Figure 6. BER Performance of Pre-ZF Equalization Based MIMO System with Implementation of 2D Median Filtering and 64 QAM Digital Modulation Schemeson Frame Sequential Video Frames(180th Frame of Each of Five Locations 12, L1, M, R1 and R2)

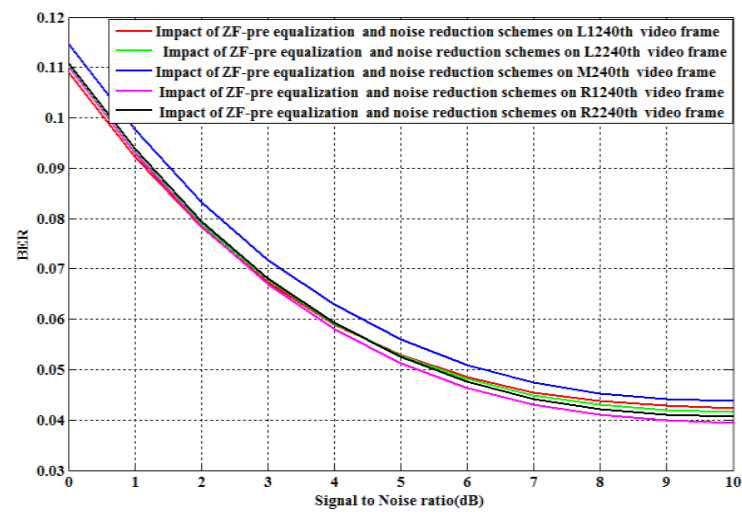

Figure 7. BER Performance of Pre-ZF Equalization Based MIMO System With Implementation Of 2D Median Filtering And 64 QAM Digital Modulation Schemeson Frame Sequential Video Frames (240th Frame Of Each Of Five Locations 12, L1, M, R1 And R2) 


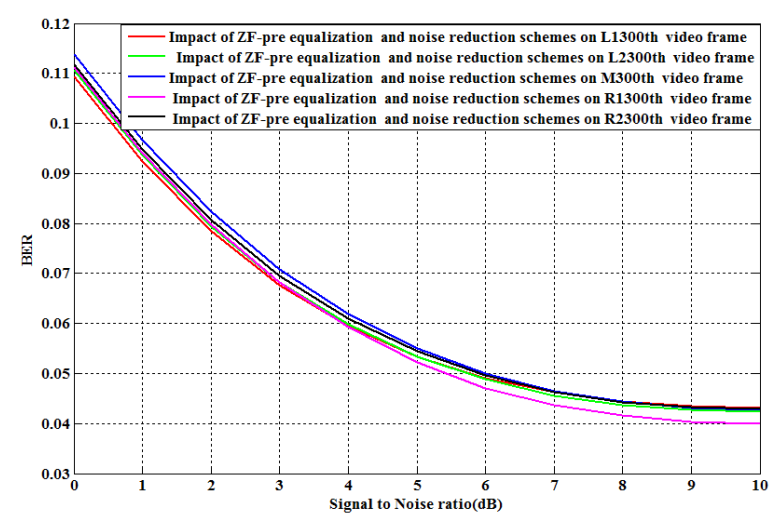

Figure 8. BER Performance of Pre-ZF Equalization Based MIMO System with Implementation of 2D Median Filtering and 64 QAM Digital Modulation Schemeson Frame Sequential Video Frames(300th Frame of Each of Five Locations I2, L1, M, R1 and R2)

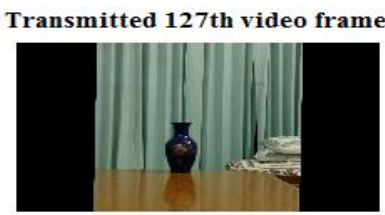

Impulse and Gaussian Noise contaminated 127th video frame

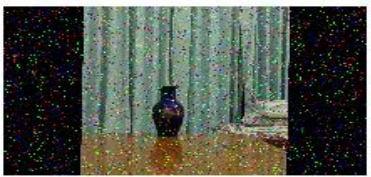

Retrieved 127 th video frame

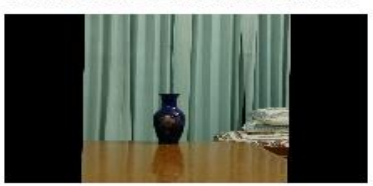

Figure 9. Uncoded System Performance Indicator for aTypically Assumed 127th Video Frame Captured with Smartphone Located at the Central Line
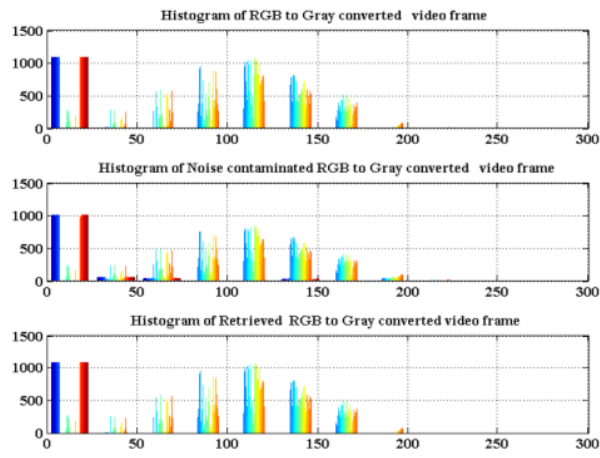

Figure 10. Histograms of RGB to Gray Converted 127th Video Frame Captured with Smartphone Located at the Central Line and its Noisy and Retrieved Versions 


\section{Conclusions}

This paper presents a comprehensive idea on the applicability of pre-channel equalization aided precoding scheme for performance evaluation of MIMO wireless communication system in scenario of video signal transmission over flat fading channel. Based on the simulation study, it is observed that without addition of any redundant data for protection, the presently considered system shows robust performance in retrieving multiview video signal in a quite acceptable form in noisy and hostile environment. In future, the work can be extended with transmission of Orthogonal/ Quasi- Orthogonal Space Time Block encoded digitally modulated complex signals over unequal number of transmit and receive antenna system.

\section{References}

[1] L. Lucas, C. Loscos and Y. Remion, “3D Video From Capture to Diffusion”, John Wiley \& Sons, Inc., USA, (2013).

[2] F. Dufaux, B. Pesquet-Popescu and M. Cagnazzo, "Emerging Technologies for 3D video creation, coding, transmission and rendering", John Wiley \& Sons, inc., United Kingdom, ( 2013).

[3] H. Cui, C. Luo, C.W. Chen and F. Wu, "Robust Uncoded Video Transmission over Wireless Fast Fading Channel", Proceedings of IEEE International Conference on Computer Communications(INFOCOM), ( 2014), pp. 73-81.

[4] Y.S. Cho, J. Kim, W.Y. Yang and C.G. Kang, "MIMO-OFDM Wireless Communications with MATLAB, John Wiley and Sons (Asia) PTE Limited", Singapore, (2010).

[5] "OgeMarques Practical Image and Video Processing Using MATLAB", John Wiley and Sons, New Jersey, USA, (2011).

[6] A. Goldsmith, "Wireless Communications", First Edition, Cambridge University Press, United Kingdom, (2005).

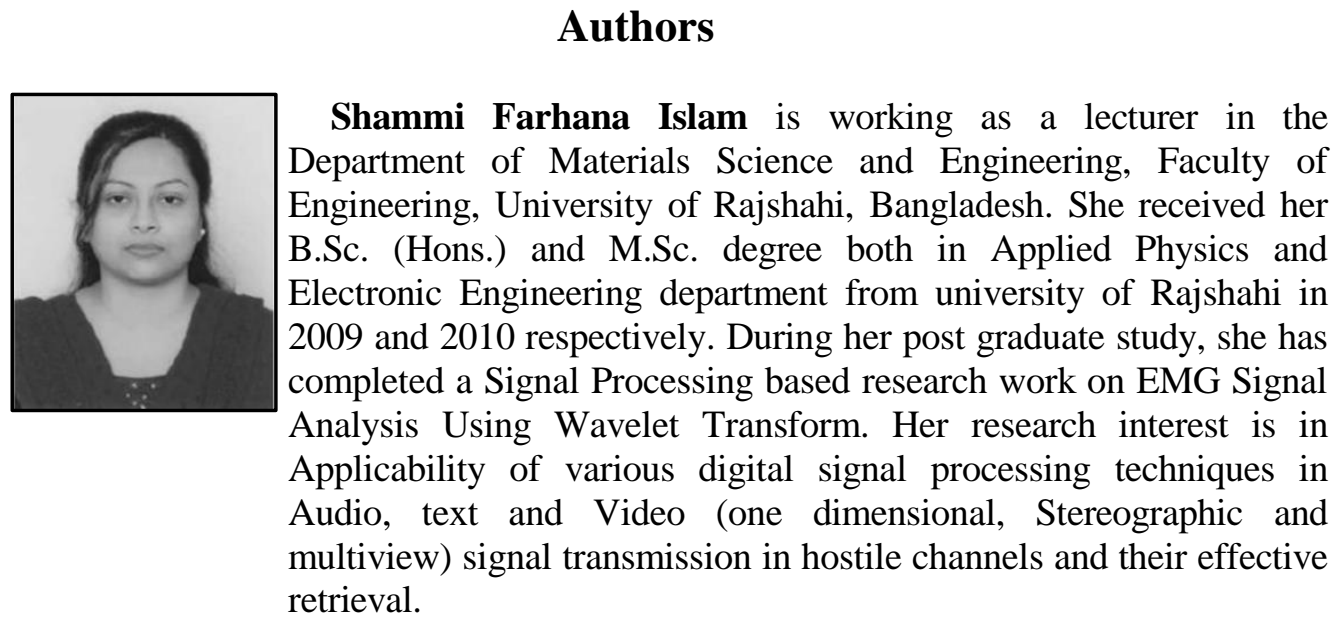

Shaikh Enayet Ullah is a Professor of the Department of Applied Physics and Electronic Engineering, Faculty of Engineering, University of Rajshahi, Bangladesh. He received US equivalent B.Sc and M.Sc degree in Physics and Electronics and Ph.D degree in Physics in 1983, 1985 and 2000 respectively. He worked as a Professor and Chairman (on deputation) in the Department of Information and Communication Engineering, University of Rajshahi. He is working as a member of both Editorial and Reviewer Board of various International Journals. He has published more than 70 papers in multidisciplinary fields. His main research interests include Cooperative communications, MIMO-OFDM, WiMAX and LTE-Advanced and Potential Field geophysical data inversion. 


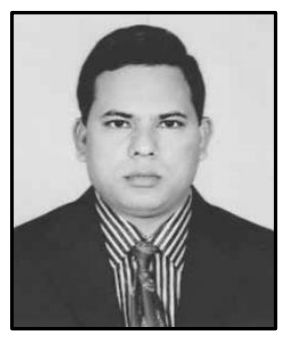

Md. Mahbubar Rahman is working as Professor of the Department of Applied Physics, Electronics and Communication Engineering, Islamic University, Kushtia, Bangladesh. He received his B.Sc (Hons) and M.Sc degrees in Applied Physics and Electronics in 1994 and 1995 respectively from University of Rajshahi, Bangladesh. Dr. Rahman received MS (Thesis) degree in Hydrology and Environmental Sciences, University of Technology, Sydney, Australia in 2006. He received his Ph.D degree in Applied Physics, Electronics and Communication Engineering, Islamic University, Bangladesh in 2009. His research interest includes Channel coding, WiMAX, CDMA, OFDMA/OFDM, MIMO technology and Environmental Science. 
International Journal of Future Generation Communication and Networking Vol. 8, No. 6 (2015) 